Ebisu

Études japonaises

Études japonaises

48 | automne-hiver 2012

Naissance d'une revue féministe au Japon : Seitō

(1911-1916)

\title{
Quand les femmes parlent d'amour... : le discours sur l'amour dans Seitō
}

When Women Talk About Love...: the Discourse on Love in Seitō

女が恋愛を語る時—『青靼』における恋愛の言説—

\section{Tomomi Ōta}

\section{OpenEdition \\ Journals}

Édition électronique

URL : http://journals.openedition.org/ebisu/619

DOI : 10.4000/ebisu.619

ISSN : 2189-1893

Éditeur :

Institut français de recherche sur le Japon (UMIFRE 19 MAEE-CNRS), Maison franco-japonaise

\section{Édition imprimée}

Date de publication : 1 septembre 2012

Pagination : 101-118

ISSN : 1340-3656

\section{Référence électronique}

Tomomi Ōta, «Quand les femmes parlent d'amour... : le discours sur l'amour dans Seitō », Ebisu [En ligne], 48 I automne-hiver 2012, mis en ligne le 21 mai 2014, consulté le 22 décembre 2019. URL : http://journals.openedition.org/ebisu/619; DOI : 10.4000/ebisu.619 


\section{Quand les femmes parlent d'amour... Le discours sur l'amour dans Seitō}

ŌTA Tomomi 太田知美*

"Le mystère de l'amour est plus grand que le mystère de la mort " écrivait Oscar Wilde dans ses aphorismes. Depuis des millénaires, cet élément infiniment mystérieux de la vie humaine continue de faire l'objet autant d'œuvres romanesques que de poésies, le Japon au tournant du vingtième siècle ne faisant pas exception. Si pourtant une particularité existe dans cette période et dans cette contrée, c'est l'abondance des essais et des dissertations publiés par des intellectuels hommes et femmes (Butel 2011a, 2011b) sur ce lien sentimental qui unit deux individus modernes, et que l'on appelle "amour" (ai 愛, ren.ai 恋愛).

Ces mots ai et ren.ai datent de l'ère Meiji (1868-1912) dans leur acception servant à traduire le mot anglais love. Bien que ce mot love ait été traduit, dès le début de l'ère Meiji, à l'aide de différents mots tels que iro 色 (le désir charnel, la luxure), koi 恋 (l'amour, en tant que manifestations concrètes de l'amour iro) ou ai (l'amour universel), le mot ai, privilégié par les intellectuels chrétiens de la revue Jogaku zasshi 女学雑誌 (Revue féminine pour les études), en vint à acquérir le sens restreint d'amour platonique. Ensuite, pour distinguer l'amour fraternel ou amical de l'amour entre homme et femme, le mot japonais préexistant koi (dont la lecture chinoise est ren) et la traduction jusqu'alors usitée ai furent combinés pour créer le néologisme ren.ai (Saeki 1998 : 10-16 ; Yanabu 1982 : 90-96 ; Butel 2011a), « amour

* Université de Toulouse II-Le Mirail, Centre d'études japonaises. 
pur » glorifié dans «Ensei shika to josei » 厭世詩家と女性 (Le Poète las du monde et la femme, Jogaku zasshi, février 1892) par Kitamura Tōkoku 北村透谷 (1868-1894).

Le discours sur ren.ai en tant qu'amour pur loué par les premiers traducteurs de l'amour se transforma par la suite, comme nous le verrons, en un discours qui magnifie l'amour comme expérience à la fois spirituelle et sexuelle sous l'influence des écrits de la féministe suédoise Ellen Key (18491926). Nous nous proposons d'étudier dans cet article les caractéristiques de ce nouveau discours sur l'amour au début du vingtième siècle, ainsi que les écrits sur l'amour, la sexualité et le mariage par des membres de la première revue littéraire féministe, Seitō 青鞜 (Les Bas-bleus) (1911-1916).

\section{Le « soi » et l'amour}

\section{à la fin de l'ère Meiji et pendant l'ère Taishōo ${ }^{1}$}

L'une des caractéristiques des discours sur l'amour au tout début du vingtième siècle est sa mise en relation avec le concept du "soi " (self, jiko 自己). Pendant l'ère Taishō (1912-1926), le concept du soi est tout particulièrement valorisé comme "partie intégrante de l'expérience moderne au même titre que la démocratie, l'humanisme libéral, et des concepts clés comme le culte de l'instruction (kyōyō shugi 教養主義), le personnalisme (jinkaku shugi 人格主義) et la formation de soi (shīyō 修養)" (Suzuki M. 2010 : 7). La formation de soi (shīyō), concept devenu populaire à la fin de l'ère Meiji et qui évolue vers le culte de l'instruction (kyōjō shugi) pendant l'ère Taishō, vise à l'accomplissement de la personnalité (jinkaku 人格) à travers la culture (kyōyō 教養) (Tsutsui 1992 : 153-162). D’ailleurs, comme le signale Suzuki Sadami, les termes jiga 自我 et jiko utilisés abondamment pendant l'ère Taishō ${ }^{2}$ sont liés au vitalisme (seimei shugi 生命主義) de Taishō

1. Quelques sections de cet article ont été publiées dans une première version dans Ōta Tomomi (2012).

2. Les deux termes jiko et jiga au sens de "soi-même " apparaissent dans le Nippo jisho 日葡辞書 Vocabulário da Lingua do Japão (Dictionnaire japonais-portugais) publié en 1603. Comme synonyme de jico (jiko) est indiqué " mizzucara vonore" (mizukara onore 自ら己れ), qui veut dire " soi-même ». Cf. Hōyaku Nippo jisho 邦訳日葡 辞書 (Traduction japonaise du «Dictionnaire japonais-portugais »), Iwanami shoten 岩波書店, 1980, pp. 360-361. Le mot jiga est utilisé depuis l'ère Meiji pour traduire la 
qui met en avant « la vie (seimei 生命) » en tant que concept fondamental dans la vision du monde (Suzuki S. 1995 : 4-9).

Le développement de la personnalité et de soi est ainsi, tant pour les hommes que pour les femmes de l'époque, un élément essentiel pour la construction d'une personne moderne. Par exemple, pour décrire les "femmes nouvelles" (atarashii onna 新しい女) ${ }^{3}$, Hiratsuka Raichō 平塚 らいてう (1886-1971), fondatrice de la revue Seitō, utilise les termes « soi » $(j i k o)$ et « formation » $($ shūyō) :

Les " femmes nouvelles » désirent maintenant la force. Elles veulent avoir la force nécessaire à l'accomplissement de la vocation de leur soi (jiko), elles veulent la force pour apprendre de nouvelles choses, pour se cultiver $(s h \bar{u} y \bar{y})$, pour grandir et pour surmonter leurs angoisses. (Hiratsuka 1913a : 7) ${ }^{4}$.

C'est pour cette découverte et cette construction de soi que l'amour joue un rôle primordial. Hiratsuka Raichō avance l'idée, notamment avec ses traductions de textes de la féministe suédoise Ellen Key, que l'amour, spirituel et sexuel, est intrinsèque au développement personnel des femmes.

Selon Key, dans l'amour, " les deux âmes partagent le plaisir sensuel et la sensualité leur procure le plaisir qui fait grandir l'âme " (Hiratsuka 1913 c : 132), et " pour que la vertu de l'amour parfait soit préservée, il faut que l'union des deux soit accomplie par la volonté des corps et des âmes " (Hiratsuka 1914 : 87). Par ailleurs, suivant la théorie de l'évolution, Key disserte sur l'amour en termes d' " amélioration " et de "développement " (Hiratsuka 1913b : 81) :

L'amour est devenu, comme je l'ai dit précédemment, une grande force spirituelle dont le génie est comparable à n’importe quelle autre force créatrice pour la formation

notion philosophique du moi (self, ego, Ich), et le terme jiko en tant que traduction du " soi », concept opposé à «l'autre " (self/other, egolalter ego, Selbst/Ander). Cf. Iwanami tetsugaku shiso jiten 岩波哲学・思想事典 (Dictionnaire Iwanami de la philosophie et de la pensée), Iwanami shoten, 2006, p. 607 et p. 620, et Nihon kokugo daijiten 日本国語 大辞典 (Grand dictionnaire de la langue japonaise), Shōgakukan 小学館, 1993, t. IX, p. 388 et p. 470 .

3. Voir les articles de Christine Lévy et de Marion Saucier dans le présent dossier.

4. Raichō cite son article sur les nouvelles femmes publié dans Chāō kōrōn 中央公論 en janvier 1913. 
du caractère. [...] L'amour doit donner la vie, [...] de nouvelles valeurs. L'amour doit enrichir l'humanité à travers ceux qui s'aiment. (Hiratsuka 1913b : 76) ${ }^{5}$.

D’après Suzuki Sadami, les idées avancées par Ellen Key ont eu, comme les pensées d'Ernst Haeckel (1834-1919), d'Henri Bergson (1859-1941), de William James (1842-1910) et de Piotr Kropotkine (1842-1921), une influence déterminante sur le vitalisme de Taishō. Suzuki pense que les idées féministes de l'ère Taishō faisaient partie du vitalisme, et que l'amour était alors considéré comme une " manifestation de la "vie" " (Suzuki S. 1995: 5-11).

Se souvenant de l'époque de la revue Seitō, Raichō écrit dans son autobiographie un passage qui montre bien le lien entre le soi et l'amour :

Les femmes (josei 女性), autrefois déniées, n'étaient considérées que comme des "femelles » (onna おんな) $)^{6}$ et non comme des êtres humains. Mais les femmes, telles que nous les avons reconnues, se doivent d'être des êtres humains à part entière. Alors la recherche de notre ego (jiga), à commencer par tout ce qui concerne le soi $(j i k o)$ - exigence de soi, développement de soi et construction de soi - s'est ensuite élargie vers autrui par l'amour (ren.ai), porté aux amoureux, aux maris et aux enfants. (Hiratsuka [1971-1973] 1992 : 288).

L'amour est ainsi considéré comme une expression de soi et un dispositif qui permet aux femmes de se développer et d'atteindre leur vrai "soi » (Suzuki M. 2010 : 7-14).

Les traductions d'écrits d'Ellen Key par Raichō, publiées dans Seitō entre janvier 1913 et décembre 1914, semblent avoir influencé le concept de l'amour chez les membres et les lectrices de Seitō ainsi que chez les intellectuels masculins de l'ère Taishō. C'est par exemple le cas des textes (non

5. L'amour est non seulement considéré pour la poursuite du bonheur individuel, mais aussi pour la conservation et l'amélioration de la qualité de l'espèce. L'idéologie eugéniste est ainsi présente chez Key : le choix de meilleurs partenaires de mariage rendu possible par l'amour libre (la sélection naturelle selon le darwinisme) contribue à l'amélioration de l'espèce.

6. Le sens courant du terme onna, transcrit habituellement à l'aide du caractère chinois 女, est " une femme ». Hiratsuka Raichō ayant ici transcrit le mot en syllabaire hiragana おんな pour désigner ces femmes d'autrefois qui n'étaient pas considérées comme égales aux hommes, ni même comme des êtres humains, nous avons choisi de le traduire par «femelles». 
fictionnels) sur l'amour, la sexualité et le mariage de Nishizaki (Ikuta) Hanayo 西崎(生田)花世 (1888-1970), d’Ueno Yō 上野葉 (1886-1928), d'Okada Yuki 岡田ゆき (1895-1966), de Yasuda (Harada) Satsuki 安田(原 田)泉月 (1887-1933) et de Yamada Waka 山田わか (1879-1957) publiés dans Seitō entre 1914 et 1915.

Ce qui a surtout influencé les intellectuels masculins de l'ère Taishō, c'est l'idée de "l'union de l'âme et du corps (dans l'amour) » (reiniku itchi 霊 肉一致), et celle du 《mariage d'amour " (ren.ai kekkon 恋愛結婚), comme c'est le cas chez Kuriyagawa Hakuson 㴻川白村 (1880-1923), dont le livre Kindai no ren.ai kan 近代の恋愛観 (La conception moderne de l'amour) publié en 1922 est devenu un best-seller ${ }^{7}$. De fait, les publications de livres sur l'amour (ren.ai) se multiplient pendant l'ère Taishō. Nous nous contenterons ici d'indiquer le nombre annuel d'ouvrages publiés en nous référant à la liste de Kanno qui recense les livres dont le titre contient "ren.ai » et quelques autres livres importants sur l'amour (Kanno 2001 : 23-25) :

\begin{tabular}{|c|c|l|l|}
\hline Année & $\begin{array}{c}\text { Nombre } \\
\text { d'ouvrages }\end{array}$ & Quelques titres significatifs & Auteurs \\
\hline 1912 & 2 & & La chasteté entre homme et femme \\
\hline 1915 & 1 & Signification sociale de l'amour & Yamada Waka \\
\hline 1920 & 1 & Le début de l'amour et de la conscience* & Kurata Hyakuzō \\
\hline 1921 & 4 & $\begin{array}{l}\text { La conception moderne de l'amour } \\
\text { L'amour usurpateur sans scrupule }\end{array}$ & $\begin{array}{l}\text { Kuriyagawa Hakuson } \\
\text { Arishima Takeo }\end{array}$ \\
\hline 1922 & 4 & Amour partagé entre homme et femme & Ishihara Jun \\
\hline 1923 & 6 & Révolution de l'amour & Yamamoto Senji \\
\hline 1924 & 10 & $\begin{array}{l}\text { Traité sur la valeur de l'amour } \\
\text { Création de l'amour }\end{array}$ & $\begin{array}{l}\text { Ishihara Jun } \\
\text { Takamure Itsue }\end{array}$ \\
\hline 1925 & 6 & $\begin{array}{l}\text { Monogamie ou amour libre } \\
\text { Amour, mariage, chasteté }\end{array}$ & $\begin{array}{l}\text { Kurata Hyakuzō } \\
\text { Mushanokōji Saneatsu }\end{array}$ \\
\hline 1926 & 4 &
\end{tabular}

* Recueil d'articles parus entre 1912 et 1920

7. Cet ouvrage est d'abord paru en feuilletons dans le quotidien Asahi shinbun 朝日 新聞, entre le 30 septembre et le 29 octobre 1921 (Kanno 2001 : 195). 
Notons aussi que, parallèlement au débat intellectuel sur l'amour, les médias se sont passionnés pour des affaires d'amour et les doubles suicides. D'un point de vue statistique, le nombre de suicides n'a pas particulièrement augmenté pendant l'ère Taishō $\overline{ }^{8}$, mais les médias leur ont consacré plus de pages qu'auparavant, en s'intéressant notamment aux doubles suicides de gens célèbres et d'aristocrates. À titre d'exemples, nous pouvons citer l'affaire Shiobara (Shiobara jiken 塩原事件), tentative de double suicide qui impliqua Hiratsuka Raichō et Morita Sōhei 森田草平 (1908), l'affaire Hikagejaya (Hikagejaya jiken 日蔭茶屋事件), tentative d'homicide d'Ōsugi Sakae 大杉栄 par Kamichika Ichiko 神近市子 à la suite de leur relation triangulaire avec Itō Noe 伊藤野枝 (1916), l'affaire Byakuren (Byakuren jiken 白 蓮事件), fuite d'amoureux de la poétesse mariée Yanagihara Byakuren 柳原 白蓮 (cousine de l'empereur Taishō) avec son amant Miyazaki Ryūsuke 宮 崎龍介 (1921), journaliste et activiste social, et le double suicide d'Arishima Takeo 有島武郎 et de la journaliste Hatano Akiko 波多野秋子 (1923).

On observe ainsi une prolifération de discours sur l'amour pendant l'ère Taishō, tant dans les revues et les livres qui analysent l'amour et le mariage que dans les articles sensationnels sur les affaires sentimentales et les doubles suicides.

Dans ce climat intellectuel qui génère des phénomènes médiatiques, l'amour semble devenir un élément essentiel à la découverte et au développement de soi pour les auteures publiant dans Seitō. Nous nous proposons pour cette raison d'examiner les textes non fictionnels sur l'amour, publiés dans cette revue.

\section{Le « soi » et l'amour chez Nishizaki Hanayo}

Nishizaki (Ikuta) Hanayo, romancière et poétesse, est née en 1888 dans le département de Tokushima. Après des études à l'école secondaire pour filles, elle commence à envoyer ses textes à la revue Joshi bundan 女子文壇 (Le monde littéraire des femmes) qui publie des œuvres de jeunes auteures ou de lectrices. Elle gagne la capitale, Tokyo, en 1910, à l'âge de 22 ans, et

8. À la fin de l'ère Meiji (entre 1905 et 1912), le nombre annuel de suicides pour 100000 personnes se situe entre 17 et 19. Il est de 20,9 en 1914, et de 20,5 en 1925 (Yuzawa $2010: 31$ ). 


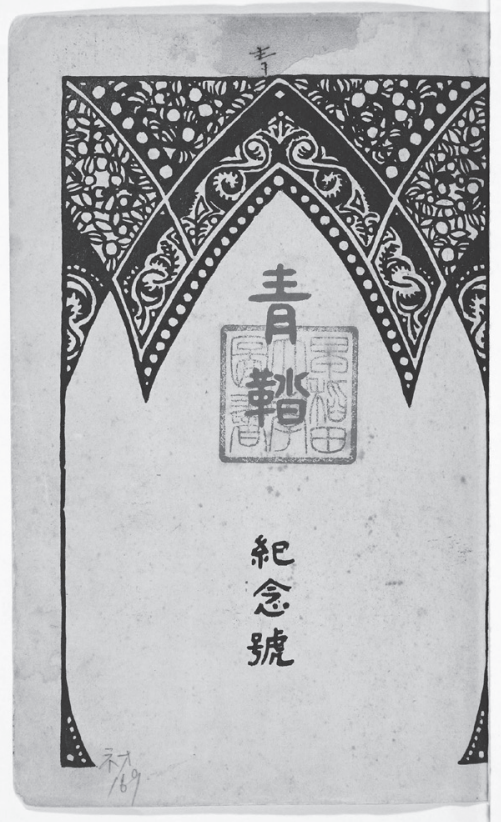

Fig. 1. Couverture de Seitō de sept. 1913 (numéro anniversaire des deux ans de la création de la revue). Bibliothèque de l'université Waseda.

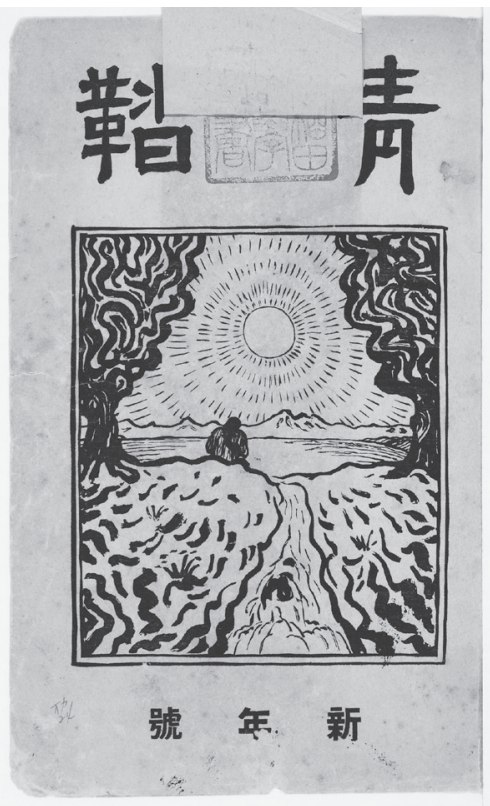

Fig. 2. Couverture du numéro de Seitō de jan. 1914, avec un dessin d'Okumura Hiroshi 奥村博. Bibliothèque de l'université Waseda.

y exerce divers métiers, tels qu'institutrice, journaliste ou serveuse, et c'est à partir de janvier 1913 qu' elle participe à la revue Seitō. Parmi les fondatrices de la revue Seitō qui ont fait leurs études à l'École d'enseignement supérieur pour jeunes filles Nihon joshi daigakkō 日本女子大学校 et les premières participantes issues d'un milieu plutôt aisé, Hanayo fait figure d'exception avec ses origines provinciales et modestes.

Elle publie un texte, présenté comme des réflexions personnelles (kansō 感想 $)^{9}$, intitulé « Ren.ai oyobi seikatsunan ni taishite» 恋愛及生活難に対して

9. Le genre kansō (réflexions personnelles) choisi par Hanayo à l'époque se situe dans la lignée de la prose de confession, développé dans la revue Joshi bundan qui publiait 
(Des difficultés de la vie amoureuse et de la vie quotidienne) dans Seitō en janvier 1914. Dans ce texte, elle confesse son besoin et son désir d'amour ; son souhait est de rencontrer un jour un homme avec qui elle pourra vivre :

Alors que la plupart des femmes possèdent concrètement les hommes, je m’aperçois que je peux posséder celui que j'aime de manière abstraite. Tandis que l'amour de la plupart des femmes est physique, le mien est métaphysique. Alors que la mort ravit ceux qui appartiennent à la terre des femmes, l'homme qui habite mon ciel baigne dans une lumière éternelle.

J'ai conscience que je suis absolument femme. Je ne saurais vivre une vie sans amour. Pour moi, vivre c'est aimer. Mais l'être aimé n’a aucune forme précise.

[...] Je suis tout entière habitée par la féminité. Je ne ressens pas la nécessité de rejeter le besoin qu'a une épouse d'être aimée. Je pense que cette conscience intelligente m’est venue tout naturellement. Je sais que si je méprisais la passion, je mépriserais ma propre vie. (Nishizaki 1914a : 73-74).

L'amour décrit dans ces deux passages est ambivalent. D’une part, Nishizaki semble ne désirer que l'amour « métaphysique » et n'avoir qu'une idée abstraite de l'objet de son amour ; d'autre part, elle ne nie pas sa passion ni son besoin d'être aimée, autrement dit l'amour "physique ", bien qu'elle n'utilise pas ici l'expression explicitement ${ }^{10}$. Sa conception de l'amour réunit ces deux aspects; son idéal est à la fois spirituel et sexuel.

Ce texte d'une quinzaine de pages est constitué de fragments de quelques paragraphes. L'omniprésence du mot « je " (watashi 私) et la répétition des verbes “penser»(omou 思う, omotta 思った, omotte iru 思っている) et " éprouver» (kanzuru 感ずる, kanjita 感じた) frappent le lecteur. Le critique littéraire Akagi Kōhei écrit qu’elle a attrapé la «maladie de la surcharge de

des œuvres de lectrices. Dans Joshi bundan, les membres du jury, presque exclusivement masculins, encourageaient leurs lectrices à conserver leur amateurisme et à s'investir dans des textes courts en prose (sanbun 散文) et non dans le roman, genre " artistique " qui requiert des techniques spécifiques. Sur la marginalisation des femmes écrivains de la fin de l'ère Meiji par rapport au genre dominant du roman, voir Odaira 2008. 10. Pendant le " débat autour de la question de chasteté » (teisō ronsō 貞操論争) dans Seitō et Hankyō 反響 (Résonance) entre 1914 et 1915, Nishizaki affirme que la raison pour laquelle elle a décidé de perdre sa virginité n'était pas uniquement liée aux difficultés de la vie quotidienne, mais aussi à son désir sexuel (qu'elle décrit par le mot " hystérie ») venant de son corps mature de vingt-quatre ans (Hankyō, février 1915 [Orii 1991: 13-18]). 
soi » (jiko kajū byo 自己過重病) qui se propageait dans les milieux littéraires de l'époque (Akagi [1914] 2006 : 144).

Son écriture introspective est poignante et touche profondément son lecteur, comme ce fut le cas pour le jeune écrivain et poète Ikuta Shungetsu 生田春月 (1892-1930). Il fut si fortement ému qu'il pensa avoir trouvé l'âme sœur. Il entra en contact avec elle par l'intermédiaire de son maitre, Kawai Suimei 河井酔茗, et lui demanda sa main, alors qu'ils ne s'étaient encore jamais rencontrés, dans une lettre d'amour d'une vingtaine de pages :

J'ai saisi la beauté de votre esprit. Et comme je suis convaincu que l'âme et le corps s'accordent, j'en déduis que vous êtes belle. [...]

Vous embellissez ma vie, vous me mettez du baume au cœur. Et moi je ferai s'épanouir votre « moi » $(g a$ 我), je vous rendrai plus femme que maintenant, et j'essuierai vos larmes. [...] Comment un être humain peut-il vivre sans aimer et sans être aimé ? Vous faites déjà partie de mon « ego " (jiga). Vos joies et vos souffrances sont miennes. (Nishizaki 1914c : 61-68).

Après quelques hésitations, Nishizaki Hanayo décide d'accepter cette demande en mariage en « acceptant de vivre ce que l'univers (uchū 宇宙) lui donnera à vivre » (Nishizaki 1914b : 37).

J'éprouve le désir de vivre pleinement mon « moi » (jiko) en restant derrière une personne, en devenant la racine de la vie de cette personne, les yeux de cette personne, la fleur du cœur de cette personne, en aidant le travail de cette personne. Je me suis aperçue que le sentiment de devenir une vraie épouse s'installe progressivement et doucement dans mon cœur. [...] Je suis enfin devenue une personne (bito 人). (Nishizaki 1914b : 37).

Hanayo accepte ainsi le mariage dans l'espoir de réaliser pleinement sa personnalité par l'expérience amoureuse. Son texte, comme la lettre de Shungetsu, sont très représentatifs du discours sur l'amour à cette époque : à travers l'amour, on (re)découvre et on améliore son " moi ».

\section{L'âme et le corps : trois conceptions de l'amour chez Okada Yuki, Yasuda Satsuki et Yamada Waka}

Quand Okada Yuki est devenue membre de la revue Seitō en 1912, elle avait dix-sept ans et était encore élève du lycée privé pour filles Kōran. C'est à partir de novembre 1914 qu'elle publie dans la revue ses écrits, dont 
sept textes de "réflexions personnelles " (kansō), trois textes sous formes de dialogues, deux pièces de théâtre et une nouvelle ${ }^{11}$. Dans son article de réflexions personnelles publié en novembre 1914 qui s'intitule « Kekkon ni tsuite ryōshin e » 結婚に就いて両親へ (À mes parents, à propos du mariage), elle s'oppose au mariage imposé par ses parents. C'est le premier texte qu'elle a publié dans Seitō.

Je voudrais absolument que mes parents sachent ceci avant de me forcer à me marier. Je suis convaincue que le mariage doit être décidé selon la volonté libre de la personne. [...]

Nous, les jeunes femmes d'aujourd'hui, nous aimons et nous respectons notre moi (jiko) plus que nos parents, nos frères et n'importe qui sur terre. [...]

Même si nos parents tentent de nous faire obéir, la conscience que nous avons de notre ego (jiga) nous empêche d'être manipulées comme des poupées.

Je respecte avant tout mon moi (jiko) plus que les autres. C'est pour cela que l'idée du mariage à la manière ancienne m’angoisse terriblement. (Okada 1914 : 109-110).

Elle rejette le mariage forcé et exprime son inclination pour le mariage libre au nom du soi (jiko) et de l'ego (jiga). Raichō note dans son autobiographie qu'Okada Yuki lisait des écrits d'Ellen Key à l'école privée d'anglais gérée par Yamada Kakichi 山田嘉吉, le mari de Yamada Waka, membre de Seitō (Hiratsuka 1992 : 242). La jeune lectrice d'Ellen Key a très probablement été influencée par ses idées, comme l'ont été Hiratsuka Raichō et Nishizaki Hanayo, et elle exprime ainsi l'importance du soi dans le lien matrimonial.

L'année suivante, en mai 1915, Yasuda (Harada) Satsuki publie un article intitulé "Shinsei to ningensei to ren.ai to » 神性と人間性と恋愛と (Divinité, humanité et amour). Elle a mené le « débat autour de la question de la chasteté " (teisō ronsō 貞操論争) dans Seito ${ }^{-12}$ contre Nishizaki (Ikuta) Hanayo entre 1914 et 1915, et après son mariage avec Harada Jun 原田潤 en 1915, elle a publié une nouvelle «Gokuchū no onna yori otoko ni » 獄中の女より 男に (D'une femme en prison à son homme) en juin de la même année, défendant l'avortement en cas d'impossibilité d'assumer ses responsabilités

11. Sur les œuvres littéraires d'Okada Yuki, voir par exemple Iwata 2003.

12. Voir l'article d'Isabelle Konuma dans le présent dossier [N.D.L.R.]. 
en tant que mère, ce qui a provoqué l'interdiction de vente du numéro de Seitō par les autorités. Cet article « Divinité, humanité et amour » est paru en mai, un mois avant la publication de la fameuse nouvelle.

Yasuda critique d'abord Tolstoï qui affirme que « le vrai mariage est autorisé dans le seul but d'engendrer des successeurs qui servent Dieu et l'humanité » et que "l'union entre hommes et femmes, physique ou spirituelle, est une dépravation, un vice, si elle vise seulement le plaisir individuel " (Harada 1915 : 187). Elle souligne ensuite que «c'est pour satisfaire leur désir individuel que les hommes et les femmes vivent l'expérience amoureuse, s'unissent et procréent " (Harada 1915 : 187-188) et que le but de l'amour n'est pas de servir Dieu et l'humanité. Elle exprime ensuite son idée de l'union de l'âme et du corps dans l'amour, pour conclure :

Puisqu'un être humain est doté d'une âme et d'un corps, l'enrichissement de l'âme seule ou du corps seul ne saurait être un véritable enrichissement de la sexualité. Si l'on reconnaît l'existence d'un corps et celle d'une âme, l'enrichissement de l'âme entraîne l'enrichissement du corps, et la satisfaction physique engendre la satisfaction spirituelle; c'est l'idéal de l'humanité. C'est précisément de cette manière que l'on peut mener une vie humaine enrichissante. (Harada 1915 : 188).

En somme, tandis qu'elle s'oppose à l'idée d'Ellen Key qui place au même niveau l'amélioration de l'espèce et la liberté individuelle pour l'amour, Yasuda approuve son idée de l'accord de l'âme et du corps. Ainsi assumet-elle pleinement le désir et l'instinct amoureux de chaque individu, tout en pensant que l'amour ne sera complet que lorsqu'il s'épanouira sur le plan mental et physique.

La même année, en septembre 1915, Yamada Waka publie, pendant le débat sur l'avortement (datai ronsōo 䜃胎論争), un article intitulé «Datai ni tsuite " 值胎に就て (Sur l'avortement). Ensuite, en novembre, elle écrit en réponse à la lettre d'une lectrice, «Ren.ai no jiyū to honnō » 恋愛の自由と本能 (La liberté de l'amour et l'instinct). Dans "Sur l'avortement », elle développe une pensée moralisatrice et écrit que l'avortement et la contraception sont tous les deux de grands péchés (zaiaku 罪悪). Enfin, sur l'amour, elle écrit dans « La liberté d'amour et l'instinct » :

Pour ceux qui dans l'union ne pensent qu'au désir physique, l'enfant est une entrave. En revanche, l'union spirituelle suscite le désir d'engendrer un enfant en tant que cristallisation de cette union. De plus, l'union née d'un véritable amour ne peut 
supporter les mesures désagréables prises pour empêcher le résultat naturel de celle-ci. Ces précautions nous insultent et salissent le véritable amour. (Yamada 1915: 77).

Les hommes tendent à préférer l'amour physique et les femmes désirent l'amour spirituel, mais [...] les femmes responsables des temps nouveaux doivent élever le désir des hommes vers ce niveau plus raffiné. (Yamada 1915 : 78).

Pour Yamada Waka, l'amour spirituel est noble tandis que l'amour physique est vulgaire. Alors que la plupart des participantes de Seitō revendiquent l'amour physique et placent l'âme et le corps sur le même plan, la conception de Yamada Waka est proche de l'amour platonique promu par les intellectuels de la revue Jogaku zasshi pendant l'ère Meiji, comme Iwamoto Yoshiharu嚴本善治 (1863-1942) ou Kitamura Tōkoku 北村透谷 ${ }^{13}$. La jeunesse douloureuse de Yamada pourrait être à l'origine de cette conception de l'amour. En effet, issue d'un milieu pauvre, elle s'est mariée aussitôt après avoir terminé ses études à l'école primaire, et a divorcé à l'âge de dix-sept ans. Ensuite, afin d'aider ses parents, elle part aux États-Unis pour travailler, mais trompée par un proxénète elle finit par travailler dans une maison close à Seattle. C'est sans doute cette expérience de l'exploitation sexuelle de son corps qui a fait naître en elle une vision de l'amour différente de celle des autres collaboratrices de Seitō, qui idéalisent l'aspect spirituel et pur de l'amour ${ }^{14}$.

\section{Ueno Yō (1886-1928) : une vision anticonformiste de l'amour}

Par rapport aux auteures que nous venons de présenter - Nishizaki (Ikuta) Hanayo, Okada Yuki, Yasuda (Harada) Satuki et Yamada Waka - Ueno Yō (1886-1928) développe des réflexions plus osées dans " "Sei" ni tsuite : Rikon seru shijū onna no omoikitta kokuhaku o yonde "「性」について— 『離婚せる四十女の思ひ切つた告白』を読んで (Sur la 《sexualité " : après avoir lu La confession hardie d'une femme divorcée de quarante ans). Ueno Yō était à l'époque professeur dans une école secondaire pour filles du département de Fukui, poste qu'elle avait obtenu après ses études à l'École normale

13. Sur ce sujet, voir par exemple Jean-Michel Butel (2011a, 2011b).

14. Elle s'échappe en 1900, trouve refuge dans un établissement d'aide aux prostituées à San Francisco, où elle se convertit au christianisme [N.D.E.]. 
supérieure de jeunes filles. Dans cet article publié en août 1914, elle fait d'abord le résumé de l'intrigue de La confession hardie d'une femme divorcée de quarante ans, publié par la romancière danoise Karin Michaëls (18721952) et traduit en japonais par Ono Hideo 小野秀雄 ${ }^{15}$, et par la suite elle exprime son opinion sur l'amour et le mariage. Tout d'abord, voici ce qu'elle déclare à propos du mariage :

Quand j'observe la situation actuelle de notre pays, je constate que non seulement la personnalité (jinkaku) des femmes est bafouée par les hommes, mais aussi que beaucoup d'hommes, bien que mariés, s'adonnent à la débauche dans les quartiers de plaisirs comme si c'était un droit. [...] Les femmes d'aujourd'hui, qui ont de l'amour-propre, peuvent-elle accepter cela ? [...] Faut-il préférer, quitte à renoncer à sa personnalité, la fausse gloire de la chasteté ? Faut-il privilégier le pain quotidien quitte à se mentir à soi-même et à mentir aux autres ? Si l'on habite chez un homme par vanité et pour la subsistance qu'il apporte, sans éprouver ni amour ni admiration pour lui, c'est une véritable escroquerie. La femme qui agit ainsi se conduit comme une prostituée. [...] Les femmes d'aujourd'hui en quête de vérité doivent avoir le courage de choisir une vie solitaire. Elles doivent être résolues à endurer l'épreuve du divorce. (Ueno 1914 : 43-44).

Elle écrit encore qu'afin qu'une femme puisse divorcer, « il lui faut avoir un savoir-faire pour pouvoir gagner sa vie" (Ueno 1914 : 44). Cette comparaison entre la prostituée et la femme qui reste mariée à un mari qu'elle n’aime plus en raison de sa dépendance économique préfigure l'opinion de l'activiste socialiste Yamakawa Kikue 山川菊栄 (1890-1980), exprimée plus d'une décennie plus tard dans son article «Keihin tsuki tokkahin toshite no onna » 景品付き特価品としての女 (La femme, un article soldé avec une prime), qui a provoqué la fameuse polémique sur l'amour avec l'anarchiste Takamure Itsue 高群逸枝 (1894-1964) ${ }^{16}$. Il y a cependant une différence

15. La traduction, avec une présentation de Kuroiwa Ruikō 黒岩涙香, a été publiée en 1913 chez Ibunkan 以文館.

16. Cet article qui développe un point de vue marxiste a été publié en janvier 1928. Yamakawa Kikue y conclut que, pour que les femmes puissent vivre sans vendre leur amour, il faut construire une société dans laquelle elles puissent vivre en gagnant leur vie au moyen d'un travail sérieux, sans être dépendantes du mariage. Takamure Itsue se lance dans la polémique avec son article "Yamakawa Kikue shi no ren.ai kan o nanzu » 山川菊栄氏の恋愛観を難ず (Critique de la vision de l’amour de Mme Yamakawa Kikue) publié dans la revue Fujin kōron 婦人公論 en mai 1928, qui réfute l'article de Yamakawa 
entre ces deux articles : Ueno Yō fait cette comparaison entre la femme mariée et la prostituée dans le cas du couple sans amour qui ne divorce pas, tandis que Yamakawa écrit dans son article que même au sein d'un couple aimant qui a contracté un mariage d'amour, tant que la femme est dépendante de son mari économiquement, elle vend son amour pour gagner sa vie, et de ce fait elle est comparable à une prostituée. Tant qu'il y a dépendance financière, il est impossible d'établir une relation amoureuse pure entre deux individus égaux. Elle réfute ainsi l'idée prônée par les intellectuels de l'ère Taishō que le mariage d'amour est meilleur que le mariage arrangé. En revanche, ce qui est frappant dans l'article d'Ueno Yō, c'est la clairvoyance avec laquelle elle analyse rationnellement aussi bien le lien amoureux que la situation financière du couple en tant qu'élément essentiel de l'institution du mariage.

Pour ce qui est de l'amour spirituel et physique, thèse chère aux membres de Seitō, elle formule son avis de la manière suivante :

N'est-il pas vrai que les personnes qui ont déjà vécu l'expérience du plaisir physique peuvent considérer l'amour et le désir sexuel comme deux questions séparées? Néanmoins il est regrettable que ces deux ne s'accordent pas. (Ueno 1914 : 42).

Avant, je pensais que le corps devait être dans tous les cas le symbole de l'âme et que l'union physique arrivait après l'union spirituelle. [...] J'étais persuadée que pour les femmes l'amour et le désir sexuel ne pouvaient pas exister séparément. Mais je sais maintenant que c'était le comble de la sottise. (Ueno 1914 : 45).

Il me semble que l'accord entre l'âme et le corps n'est qu'un rêve de vierges. (Ueno $1914: 47)$.

en développant un point de vue anarchiste. Takamure explique que la division du travail fait obstacle au véritable développement de l'amour et que dans la société marxiste cette division subsiste encore. Pour Takamure, le véritable amour se développe dans une société de fédération libre construite sur la base de la communauté villageoise où n'existe pas encore la division du travail. Yamakawa répond dans son article intitulé " Doguma kara deta yūrei »ドグマから出た幽霊 (Un fantôme né d'un dogme), publié en juin 1928 dans la même revue, et critique l'idéalisation par Takamure de la vie des femmes à la campagne. La dernière réfutation de Takamure dans «Fumareta inu ga hoeru » 踏まれ た犬が㭈える (Un chien piétiné aboie), publié le mois suivant, n’est que le plaidoyer de l'anarchisme. La polémique s'élargit à celle qui oppose le marxisme et l'anarchisme et, pour finir, le cœur du débat n'est plus vraiment la question de l'amour. 
Ainsi Ueno Yō n'accepte-t-elle pas sans examen la conception de l'amour en vogue à cette époque, qui prônait l'idée de l'accord entre l'âme et le corps. Elle admet en revanche que l'amour spirituel et le désir sexuel peuvent exister indépendamment l'un de l'autre, et que le désir physique peut naître même avant les sentiments amoureux ${ }^{17}$. Voici un bel exemple d'audace et d'anticonformisme pour une femme de cette époque. Aussi ses idées originales, différentes de celles d'autres contributrices de la revue, occupent-elles une place particulière au sein de Seitō.

L'amour a ainsi été analysé dans Seitō en tant qu'élément essentiel pour le développement du soi et de l'ego, et en relation avec l'idée de l'accord entre l'âme et le corps. Le discours sur l'amour dans cette revue correspondait donc largement aux traits distinctifs du discours sur l'amour de l'ère Taishō caractérisé par la croyance en la supériorité de l'amour et du mariage d'amour. Comme nous venons de le voir, Nishizaki (Ikuta) Hanayo considère son mariage d'amour comme le meilleur moyen de réaliser pleinement son " moi ", Okada Yuki refuse le mariage forcé qui va à l'encontre de son ego, et Yasuda (Harada) Satsuki croit à l'idée de l'accord entre l'âme et le corps. La vision de l'amour chez Yamada Waka se rapproche de celle de l'amour platonique chez les intellectuels de l'ère Meiji, influencés par le christianisme. Enfin, Ueno Yō propose un point de vue original sur le mariage et sur l'accord entre amour spirituel et sexuel. Sa vision désenchantée du mariage et son affirmation du désir sexuel détaché des sentiments amoureux font d'elle une figure d'exception parmi les intellectuelles japonaises de l'époque.

Plus tard, aux alentours de 1928, pendant les premières années de l'ère Shōwa, on constate une nouvelle vogue de discours sur l'amour, plus réalistes et parfois plus radicaux que ceux de l'ère Taishō, dans certaines revues féminines, littéraires ou généralistes, telles que Nyonin geijutsu 女人芸術

17. Sur ce point Ogura Seizaburō 小倉清三郎 (1882-1941) rejoint les analyses d'Ueno Yō dans son article "Seiteki seikatsu to fujin mondai " 性的生活と婦人問題 (La vie sexuelle et la question féminine) paru dans Seitō en décembre 1914. Il s'agit d'un sexologue qui a contribué à Seitō avec trois articles. 
(Femmes artistes), Fujin kōron 婦人公論 (Débat public des femmes) et Fujin sensen 婦人戦線 (Le Front des femmes), et les polémistes féministes soulèvent des débats sur l'importance de l'indépendance économique des femmes dans le mariage. La vision idéaliste de l'amour au sein de Seitō n'est cependant pas complètement sans lien avec la vision réaliste et désabusée de l'amour de l'ère Shōwa. De même que nous avons observé un lien entre Ueno Yō et Yamakawa Kikue, nous trouvons une certaine filiation entre les conceptions de Seitō et le discours sur l'amour une décennie plus tard. Le débat sur l'amour engagé par les intellectuels masculins de l'ère Meiji, poursuivi par ceux de l'ère Taishō et par les rédactrices de Seitō, prendra une nouvelle tournure dans l'acte suivant.

\section{Bibliographie}

AKAGI Kōhei 赤城林平 [1914] 2006 « Sangatsu no hyōron » 三月の評論 (Critique du mois de mars), [éd.originale : Hototogisu ホトトギス, avril 1914], repris dans Bungei jihyō taikei Taishō hen 文芸時評大系大正編 (Bibliothèque complète de critique littéraire. Ėre Taishō), Tokyo,

Yumani shobō ゆまに書房.

\section{BUTEL Jean-Michel 2011a}

«Forger un amour moderne : petite histoire du mot ren.ai », in GALAN Christian et LOZERAND Emmanuel (dir.), La famille japonaise moderne (18681926). Discours et débats, Arles, Picquier, pp. 335-346.
BUTEL Jean-Michel 2011b

« Des couples aimants pour une nation moderne », in GALAN Christian et LOZERAND Emmanuel (dir.), La famille japonaise moderne (1868-1926). Discours et débats, Arles, Picquier, pp. 361-378.

HARADA Satsuki 原田皇月 1915 "Shinsei to ningensei to ren.ai to » 神性と人間性と恋愛と (Divinité, humanité et amour), Seitō, mai : 180-188.

HIRATSUKA Raichō 平塚らいてう 1913a «Ren.ai to kekkon : Eren Kei cho» 恋愛と結婚一一レン•ケイ著 (Amour et mariage : l'ouvrage d'Ellen Key), Seitō, janvier, annexe : 1-19. 


\section{HIRATSUKA Raichō 1913b}

«Seiteki dōtoku hatten no katei : Eren

Kei » 性的道徳発展の過程——エン・ケイ

(Évolution de la morale sexuelle : Ellen

Key), Seitō, août : 72-86.

HIRATSUKA Raichō $1913 c$

«Ren.ai no shinka : Eren Kei » 恋愛の進化 ——レン•ケイ (Évolution de l'amour :

Ellen Key), Seitō, octobre : 126-133.

\section{HIRATSUKA Raichō 1914}

«Ren.ai no jiyū : Eren Kei » 恋愛の自由 —エレン•ケイ (Liberté de l'amour :

Ellen Key), Seitō, juillet : 80-91.

HIRATSUKA Raichō [1971-1973] 1992

Genshi, josei wa taiyō de atta 元始、

女性は太陽であった (À l'origine, la femme était le soleil), Tokyo [éd. originale :

Ōtsuki shoten 大月書店], éd. de poche :

Kokumin bunko 国民文庫.

IWATA Nanatsu 岩田ななつ 2003

Bungaku to shite no Seitō 文学としての

『青鞜』(Seitō en tant que littérature),

Tokyo, Fuji shuppan 不二出版.

KANNO Satomi 菅野聡美 2001

Shōhi sareru ren.ai-ron : Taishō chishikijin to sei 消費される恋愛論—大正知識人

と性 (La vogue des discours sur l'amour : les intellectuels de l'ère Taishō et la sexualité), Tokyo, Seikyūsha 青弓社.

NISHIZAKI Hanayo 西崎花世 1914a "Ren.ai oyobi seikatsunan ni taishite» 恋愛及生活難に対して (Des difficultés de la vie amoureuse et de la vie quotidienne), Seitō, janvier : 70-84.
NISHIZAKI Hanayo1914b

«Makoto o shitaite » 真をしたいて

(Aimer la vérité), Seitō, mars : 25-49.

NISHIZAKI Hanayo 1914c

«Etaru "inochi"» 得たる「いのち」

(«La vie » que j'ai obtenue), Seitō, mai : 58-71.

ODAIRA Maiko 小平麻衣子 2008 Onna ga onna o enjiru. Bungaku, yokubō, shōhi 女が女を演じる—文学、欲望、消費 (La femme dans le rôle de la femme: littérature, désir et consommation), Tokyo, Shin.yōsha 新曜社.

OKADA Yuki 岡田ゆき1914

«Kekkon ni tsuite ryōshin e » 結婚に 就いて両親へ (À mes parents, à propos du mariage), Seitō, novembre : 109-110.

ORII Miyako 折井美耶子 1991

Sei to ai o meguru ronsō 性亡愛を めぐる論争 (Débats sur le sexe et l'amour), Tokyo, Domesu shuppan ドメス出版.

\section{ŌTA Tomomi 2012}

«Devenir écrivain/devenir femme/ devenir soi : l'écriture et l'amour dans les textes littéraires de Seitō (Les Bas-bleus) », in ANDRO-UEDA Makiko et BUTEL Jean-Michel (dir.), Japon Pluriel 9, Arles, Philippe Picquier.

SAEKI Junko 佐伯順子 1998 Iro to ai no hikaku bunka-shi 色亡愛の 比較文化史 (Histoire culturelle comparée de la luxure et de l'amour), Tokyo, Iwanami shoten 岩波書店. 
SUZUKI Michiko 2010

Becoming Modern Women,

Stanford, Stanford University Press.

SUZUKI Sadami 鈴木貞美 (dir.) 1995

Taishō seimei shugi to gendai 大正

生命主義と現代 (Le vitalisme de Taishō

et l'époque contemporaine), Tokyo,

Kawade shobō shinsha 河出書房新社.

TSUTSUI Kiyotada 筒井清忠 1992

« Kindai Nihon no kyōyō shugi to shūyō

shugi » 近代日本の教養主義と修養主義

(Le culturalisme et la formation de soi

dans le Japon moderne), Shisō 思想,

février: 151-174.

UENO Yō 上野葉 1914

" "Sei" ni tsuite : Rikon seru shijū onna

no omoikitta kokuhaku o yonde »

「性」について——離婚せる四十女の

思ひ切つた告白』を読んで

(Sur « la sexualité » : après avoir lu

La confession hardie d'une femme

divorcée de quarante ans),

Seitō, août : 30-49.

YAMADA Waka 山田わか 1915

« Ren.ai no jiyū to honnō » 恋愛の自由と 本能 (La liberté en amour et l'instinct),

Seitō, novembre : 72-79.

YAMAKAWA Kikue 山川菊栄 [1928] 1982

"Keihin tsuki tokkahin toshite no onna »

景品付き特価品としての女

(La femme, un article soldé avec une prime), [éd. originale : Fujin kōron

婦人公論, janvier 1928], recueilli dans

Yamakawa Kikue shū 山川菊栄集 (Euvres

de Yamakawa Kikue), t. V, Tokyo,

Iwanami shoten 岩波書店, pp. 2-8.
YANABU Akira 柳父章 1982

Hon.yakugo seiritsu jijō 翻訳語成立事情

(La formation du vocabulaire japonais

issu de la traduction de termes

étrangers), Tokyo, Iwanami shoten

岩波書店.

YUZAWA Yasuhiko 湯沢雍彦 2010

Taishō ki no kazoku mondai 大正期の

家族問題 (Problèmes familiaux de l'ère

Taishō), Tokyo, Mineruva shobō

ミネルヴァ書房. 